\title{
XLI. On certain diffraction fringes as applied to micrometric observations
}

\section{N. G. Filon M.A.}

To cite this article: L. N. G. Filon M.A. (1899) XLI. On certain diffraction fringes as applied to micrometric observations, Philosophical Magazine Series 5, 47:288, 441-461, DOI:

10.1080/14786449908621284

To link to this article: http://dx.doi.org/10.1080/14786449908621284

曲 Published online: 08 May 2009.

Submit your article to this journal $\lceil\pi$

Џ Article views: 3

Q View related articles $\asymp$ 
Fig. 3.-Instantaneous Form of Wave-Train for $k=\cdot 3 / \pi$, whence $R^{\prime \prime} / R^{\prime}=1 \cdot 054$.

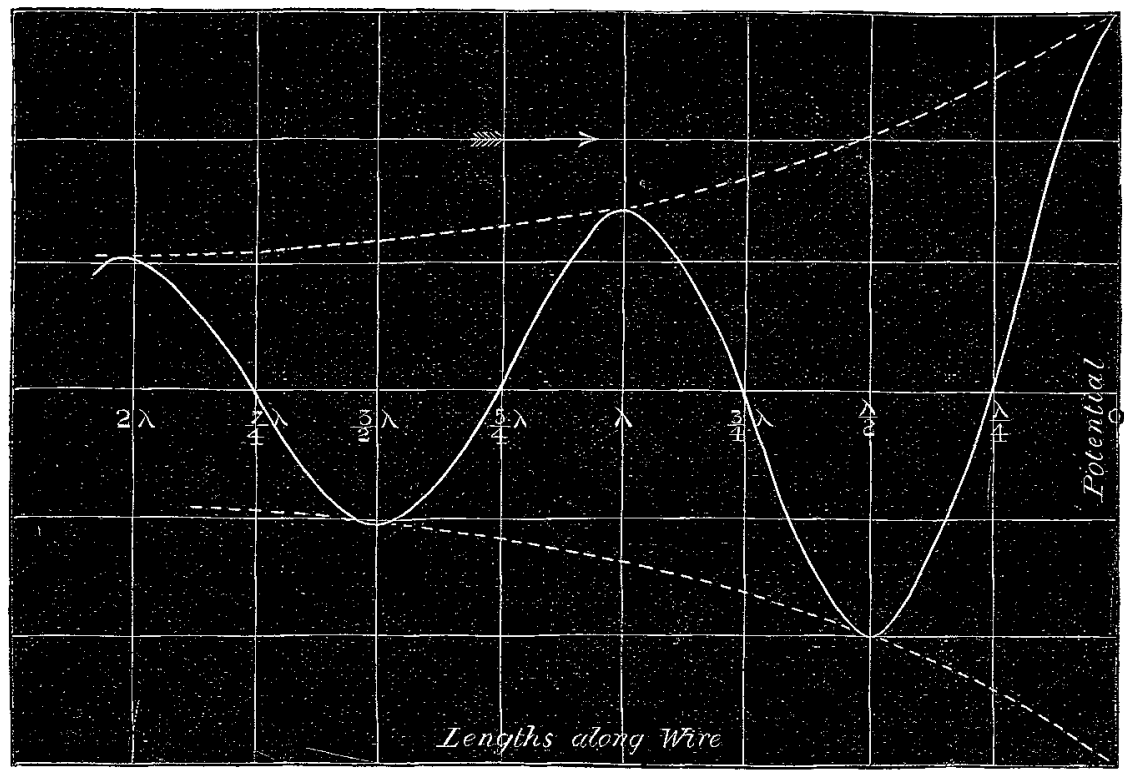

the experiments just referred to the frequency was $35 \times 10^{\circ}$ per second, and $R^{\prime} / R$ became $31 \cdot 6$. Hence $R^{\prime \prime} / R$ has the value $31 \cdot 6 \times 1 \cdot 054=33.3$ nearly. Thus, writing $e^{-\mathrm{R}^{\prime \prime} x / 2 \mathrm{~L} o}$ for the attenuator of the waves along the wires instead of * $e^{-\mathrm{R}^{\prime} x^{\prime} / 2 \mathrm{~L} v}$ increases the index by about five and a half per cent., and so brings it by that amount nearer to the value determined experimentally.

Univ. Coll., Nottingham, Nov. $29,1898$.

XLI. On certain Diffraction Fringes as applied to Micrometric Observations. By L. N. G. FILoN, M.A., Dernonstrator in Applied Mathematics and Fellow of University College, London†.

1. TWHE following paper is largely criticism and exten1 sion of Mr. A. A. Michelson's memoir "On the Application of Interference Methods to Astronomical Observations," published in the Phil. Mag. vol. xxx. p. 256, March 1891.

* See Equation (2) p. 301, Phil. Mag. Sept. 1898.

+ Communicated by the Physical Society : read November 25, 1898. 
Light from a distant source is allowed to pass through two thin parallel slits. The rays are then focussed on a screen (or the retina of the eye) and interference-fringes are seen. If the distant source be really double, or extended, the fringes will disappear for certain values of the distance between the slits. This distance depends on the angle subtended by the two components of the double source or the diameter of the extended source.

Mr. Michelson, however, in obtaining his results treated the breadth of the slits as small compared with the wavelength of light and their length as infinite. This seems unjustifiable $\dot{a}$ priori. The present investigation takes the dimensions of the slits into account.

2. Suppose we have an aperture or diaphragm of any shape in a screen placed just in front of the object-glass of a telescope (fig. 1).

Fig. 1.

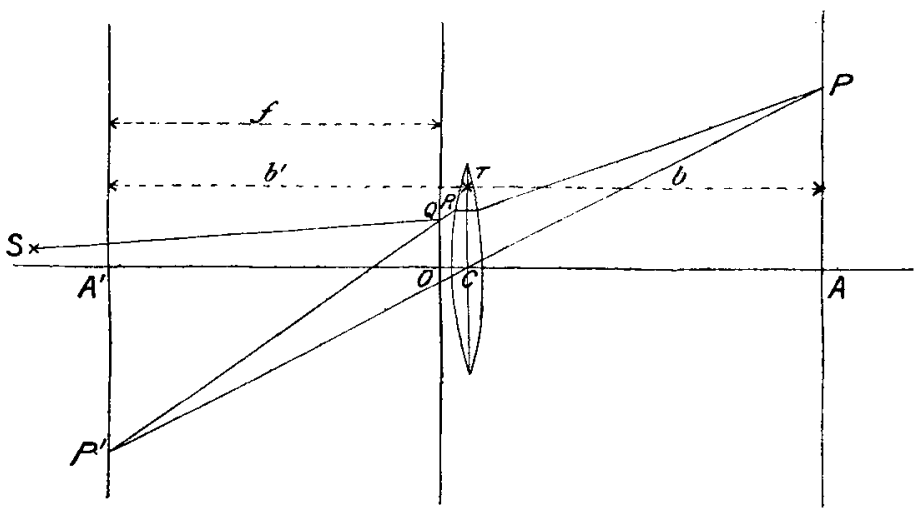

Let the axis of the telescope be the axis of $z$. Let the axes of $x$ and $y$ be taken in the plane of the diaphragm OQ perpendicular to and in the plane of the paper respectively. Let $S$ be a source of light whose coordinates are $U, V, W$. Let $\mathrm{Q}$ be any point in the diaphragm whose coordinates are $(x, y)$. Let AP be a screen perpendicular to the axis of the telescope, and let $(p, q)$ be the coordinates of any point $\mathrm{P}$ on this screen. Let $\mathrm{A}^{\prime} \mathrm{P}^{\prime}$ be the conjugate image of the screen AP in the object-glass.

Let $b=$ distance of centre $\mathrm{C}$ of lens from screen AP.

$$
\begin{aligned}
& b^{\prime}=\quad " \quad " \quad, \quad \text { image of screen AP. } \\
& f=\text { distance of diaphragm } 0 \text { "Q from plane } \mathrm{A}^{\prime} \mathrm{P}^{\prime} \text {. }
\end{aligned}
$$

Then if, as is usual, we break up a wave of light coming 
as applied to Micrometric Observations.

from $S$ at the diaphragm, the secondary wave due to the disturbance at $Q$ would have to travel along a path QRTP in order to reach a point $\mathrm{P}$ on the screen, being regularly refracted.

But since $\mathrm{P}^{\prime}$ is the geometrical image of $P$, all rays which converge to $\mathrm{P}$ (i.e. pass through $\mathrm{P}$ ) after refraction, must have passed through $\mathrm{P}^{\prime}$ before refraction, to the order of our approximation. $\mathrm{P}^{\prime} \mathrm{Q}$.

Hence the ray through $Q$ which is to reach $P$ must be

Moreover, $\mathrm{P}$ and $\mathrm{P}^{\prime}$ being conjugate images the change of phase of a wave travelling from $\mathbf{P}^{\prime}$ to $P$ is constant to the first approximation and independent of the position of $Q$.

Now the disturbance at $\mathrm{P}$ due to an element $d x d y$ of the diaphragm at $Q$ is of the form

$$
\frac{\mathrm{A} d x d y}{b \lambda} \sin \frac{2 \pi}{\lambda}\left(\frac{\lambda t}{\tau}-\mathrm{SQ}-\mathrm{QR}-\mu . \mathrm{RT}-\mathrm{TP}\right),
$$

where $\lambda$ is the wave-length, $\tau$ is the period, $A$ is a constant, $\mu$ is the index of refraction of the material of the lens, and $b$ is put instead of $\mathrm{QP}$ outside the trigonometrical term, because the distance of the lens from the diaphragm and the inclination of the rays are supposed small.

But $\quad \mathrm{P}^{\prime} \mathrm{Q}+\mathrm{QR}+\mu \mathrm{RT}+\mathrm{TP}=$ constant for $\mathrm{P}$.

Therefore the disturbance

But

$$
=\frac{\mathrm{A} d x d y}{b \lambda} \sin \frac{2 \pi}{\lambda}\left(\frac{\lambda t}{\tau}-\mathrm{SQ}+\mathrm{P}^{\prime} \mathrm{Q}-\text { const. }\right) .
$$

$$
\begin{aligned}
\mathrm{SQ}^{2} & =(x-\mathrm{U})^{2}+(y-\mathrm{V})^{2}+\mathrm{W}^{2} . \\
\mathrm{P}^{\prime} \mathrm{Q}^{2} & =\left(x+\frac{b^{\prime}}{b} p\right)^{2}+\left(y+\frac{b^{\prime}}{b} g\right)^{2}+f^{2} .
\end{aligned}
$$

Now in practice $x, y, p, q$ are small compared with $b^{\prime}, b, f$, or $\mathrm{W} ; \mathrm{U}$ and $\mathrm{V}$ are small compared with $\mathrm{W}$. Neglecting terms of order $\mathrm{U}^{3} / \mathrm{W}^{3}, x \mathrm{U}^{2} / \mathrm{W}^{3}$, \&c., we find

$$
\mathrm{SQ}=\sqrt{\mathrm{U}^{2}+\mathrm{V}^{2}+\mathrm{W}^{2}}-\frac{\mathrm{U}}{\mathrm{W}} x-\frac{\mathrm{V}}{\overline{\mathrm{W}}} y+\frac{1}{2} \frac{x^{2}+y^{2}}{\mathrm{~W}} .
$$

In like manner

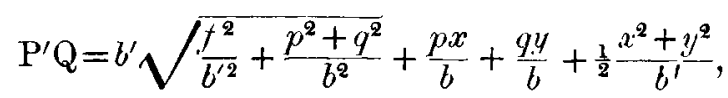

remembering that $f$ is very nearly equal to $b^{\prime}$ because the diaphragm is very close to the lens. 
441 Mr. L. N. G. Filon on certuin Diffraction Fringes air

Hence the difference of retardation measured by length in

$$
\begin{aligned}
\mathrm{P}^{\prime} \mathrm{Q}-\mathrm{SQ}=\text { const. }+\left(\frac{p}{b}+\frac{\mathrm{U}}{\mathrm{W}}\right) & x+\left(\frac{q}{b}+\frac{\mathrm{V}}{\mathrm{W}}\right) y \\
& +\frac{1}{2}\left(x^{2}+y^{2}\right)\left(\frac{1}{b^{\prime}}-\frac{1}{\mathrm{~W}}\right) .
\end{aligned}
$$

If now the geometrical image of $S$ lie on the screen AP (i.e. if the screen is in correct focus) $b^{\prime}=\mathrm{W}$ and the last term disappears.

If, however, the screen be out of focus $1 / b^{\prime}$ is not equal to $1 / \mathrm{W}$, and the term in $x^{2}+y^{2}$ may be comparable with the two others, if $t^{\prime}$ be not very great compared with $b$. Thus we see that appearances out of focus will introduce expressions of the same kind as those which occur when no lonses are used.

We will, however, only consider the case where the screen is in focus. Let $-u$ and $-v$ be the coordinates of the geometrical image of $S$; then

$$
u / b=\mathrm{U} / \mathrm{W}, \quad v / b=\mathrm{V} / \mathrm{W} .
$$

The difference of retardation measured by length in air is therefore of the form

$$
\mathrm{P}^{\prime} \mathrm{Q}-\mathrm{SQ}=\text { const. }+\frac{p+u}{b} x+\frac{q+v}{b} y .
$$

Hence the total disturbance at $P$ (integrating over the two slits) is given by the expression

$$
\begin{aligned}
\mathrm{D} & =\frac{\mathrm{A}}{b \lambda} \int_{a-k}^{a+k} d y \int_{-k}^{\boldsymbol{k}} d x \sin \frac{2 \pi}{\lambda}\left(\frac{\lambda t}{\tau}+\frac{p+u}{b} x+\frac{q+v}{b} y\right) \\
& +\frac{\mathrm{A}}{b \lambda} \int_{-a-k}^{-a+k} d y \int_{-k}^{k} d x \sin \frac{2 \pi}{\lambda}\left(\frac{\lambda t}{\tau}+\frac{p+u}{b} x+\frac{q+v}{b} y\right),
\end{aligned}
$$

where

$$
\begin{aligned}
& \text { A }=\text { a constant, } \\
& 2 a=\text { distance between centres of slits, } \\
& 2 k=\text { breadth of either slit, } \\
& 2 h=\text { length of either slit. }
\end{aligned}
$$

This, being integrated out, gives

$$
\mathrm{D}=\frac{2 \mathrm{~A} b \lambda}{\pi^{2}(p+u)(q+v)} \sin \frac{2 \pi t}{\tau} \cos \frac{2 \pi}{\lambda} \frac{q+v}{b} a \sin \frac{2 \pi}{\lambda} \frac{q+v}{b} k \sin \frac{2 \pi}{\lambda} \frac{p+u}{b} h,
$$


whence the intensity of light

$$
\mathrm{I}=\frac{4 A^{2} b^{2} \lambda^{2}}{\pi^{4}(p+u)^{2}(q+v)^{2}} \cos ^{2} \frac{2 \pi a}{b \lambda}(q+v) \sin ^{2} \frac{2 \pi k}{b \lambda}(q+v) \sin ^{2} \frac{2 \pi l}{b \lambda}(p+u) .
$$

This may be written

$$
\frac{64 \mathrm{~A}^{2} k^{2} h^{2}}{b^{2} \lambda^{2}} \cos ^{2} \frac{2 \pi \alpha}{b \lambda}(q+v) \frac{\sin ^{2} \frac{2 \pi k}{b \lambda}(q+v) \sin ^{2} \frac{2 \pi h}{b \lambda}(p+u)}{\left(\frac{2 \pi k}{b \lambda}(q+v)\right)^{2}} \frac{2 \pi h}{\left(\frac{2 \pi \lambda}{b \lambda}(p+u)\right)^{2}} \text {. }
$$

This gives fringes parallel to $x$ and $y: k$ being very small compared with $a$, the quick variation term in $v$ is

$$
\cos ^{2} \frac{2 \pi a}{b \lambda}(q+v)
$$

Consider the other two factors, namely :

$$
\frac{\sin ^{2} \frac{2 \pi k}{b \lambda}(q+v)}{\left\{\frac{2 \pi k}{b \lambda}(q+v)\right\}^{2}} \text { and } \frac{\sin ^{2} \frac{2 \pi h}{b \lambda}(p+u)}{\left\{\frac{2 \pi h}{b \lambda}(p+u)\right\}^{2}} \text {. }
$$

If we draw the curve $y=\frac{\sin ^{2} x}{x^{2}}$ (see fig. 2), we see that these factors are only sensible, and therefore their product is only sensible, for values of $p+u$ and $q+v$ which are numerically less than $b \lambda / 2 h$ and $b \lambda / 2 k$ respectively.

Fig. 2.

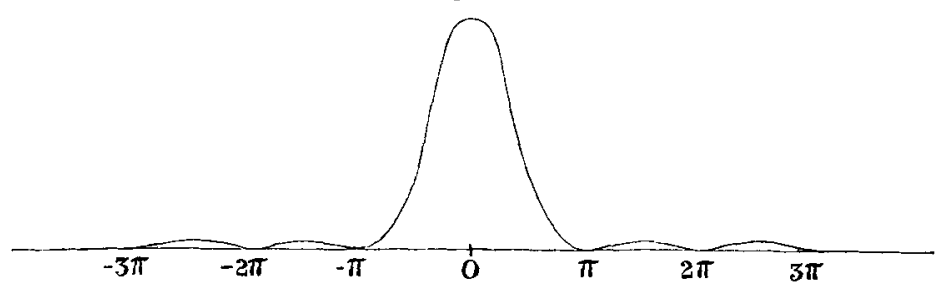

Hence the intensity becomes very small outside a rectangle whose centre is the geometrical image and whose vertical and horizontal sides are $b \lambda / k$ and $b \lambda / h$ respectively.

This rectangle I shall refer to as the "visible "rectangle of the source.

Inside this rectangle are a number of fringes, the dark lines being given by

$$
q+v=\frac{2 n+1}{4 a} b \lambda
$$

and the bright ones by $q+v=n b \lambda / 2 \alpha$. 
The successive maximum and minimum intensities do not vary with $a$. Hence, what Mr. Michelson calls the measure of visibility of the fringes, namely the quantity

$$
\frac{I_{1}-I_{2}}{I_{1}+I_{2}}
$$

where $I_{1}, I_{2}$ are successive maximum and minimum intensities, does not vary with the distance between the slits. The only effect of varying the latter is to make the fringes close up or open out. Hence for a point-source of light the fringes cannot be made to practically disappear.

3. Consider now two point-sources of light whose geometrical images are $J_{1}, J_{2}$, and draw their visible rectangles (fig. 3).

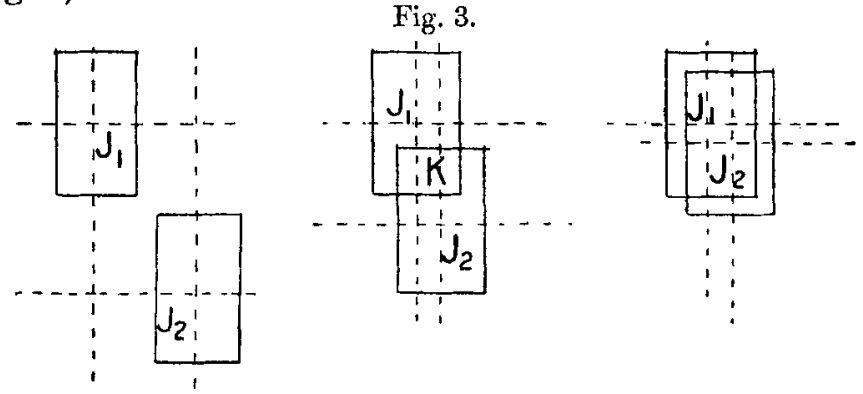

To get the resultant intensity we have to add the intensities at every point due to each source separately.

Then it may be easily seen that the following are the phenomena observed in the three cases shown in fig. 3:-

(1) The two sets of tringes distinct. Consequently no motion of the slits can destroy the fringes. In this case, however, the eye can at once distinguish between the two sources and Michelson's method is unnecessary.

(2) Partial superposition : the greatest effect is round the point $K$, where the intensities due to the two sources are very nearly equal. If $v^{\prime}-v$ be the distance between $J_{1}$ and $J_{2}$ measured perpendicularly to the slits, so that $\left(v^{\prime}-v\right) / b$ is the difference of altitude of the two stars when the slits are horizontal, then over the common area the fringe system is (a) intensified if $v^{\prime}-v$ be an even multiple of $b \lambda / 4 a,(b)$ weakened, or even destroyed, if $v^{\prime}-v$ be an odd multiple of $b \lambda / 4 a$. For in case (a) the maxima of one system are superposed upon the maxima of the other, while in case $(b)$ the maxima of the one are superposed upon the minima of the other. This common area, however, will contain only comparatively faint fringes, the more distinct ones round the 
centres remaining unaffected. We may suppose case (2) to occur whenever the centre of either rectangle lies outside the other, i. e. whenever $v^{\prime}-v>b \lambda / 2 k, u^{\prime}-u>b \lambda / 2 h, u^{\prime}-u$ being the horizontal distance between $J_{1}$ and $J_{2}$.

(3) Almost complete superposition of the visible rectangles. The fringes of high intensity are now affected. These are destroyed or weakened whenever $a$ is an odd multiple of $b \lambda / 4\left(v^{\prime}-v\right)$, provided that the intensity of one source be not small compared with that of the other.

Case (3) may be taken to occur when $v^{\prime}-v<b \lambda / 2 k$ and $u^{\prime}-u<b \lambda / 2 h$.

The smallest value of $a$ for which the fringes disappear is $l \lambda / 4\left(v^{\prime}-v\right)$.

If $v^{\prime}-v$ be very small, this may give a large value of $a$.

Now a double star ceases to be resolved by a telescope of aperture $2 r$ if $\left(v^{\prime}-v\right) / b \overline{\overline{<}} \lambda / 2 r$, and when this relation holds the smallest value of $a$ for which the fringes disappear is not less than $r / 2$, which is the greatest separation of the slits which can conveniently be used. Hence the method ceases to be available precisely at the moment when it is most needed.

(4) Mr. Michelson, in the paper quoted above, noticed this difficulty, and described an apparatus by means of which the effective aperture of the telescope could be indefinitely increased. $\mathrm{He}$ has not shown, however, that the expression for the disturbance remains of the same form, to the order of approximation taken, and he has made no attempt to work out the results when the slit is taken of finite width, as it should be.

In his paper Mr. Michelson describes two kinds of apparatus. I shall confine my attention to the second one, as being somewhat more symmetrical.

So far as I can gather from Mr. Michelson's description, the instrument consists primarily of a system of three mirrors $a, b, c$ and two strips of glass $e, d$ (fig. 4). The mirrors $a$ and $b$ are parallel, and $c, d, e$ are parallel. Light from a point $\mathrm{P}$ in one slit is reflected at $Q$ and $R$ by the mirrors $a$ and $b$, is refracted through the strip $e$, and finally emerges parallel to its original direction as $\mathrm{T} U$. Light from a point $\mathrm{P}^{\prime}$ in the other slit is refracted through the strip $d$, and reflected at $\mathbf{S}^{\prime}$ and ' $\mathrm{T}^{\prime}$ by the strips $e$ and $c$.

I may notice in passing that the strip $e$ should be half silvered, but not at the back, for if the ray $\mathrm{S}^{\prime} \mathrm{T}^{\prime}$ is allowed to penetrate inside the strip and emerge after two refractions and one reflexion, not only is a change of phase introduced, owing to the path in the glass, which complicates the analysis, 
but the conditions of reflexion, which should be the same for all four mirrors, are altered, and this changes the intensities of the two streams. We shall see afterwards that this silvering can be done without impeding the passage of the transmitted stream, as it will turn out that the two streams must be kept separate.

Fig. 4.

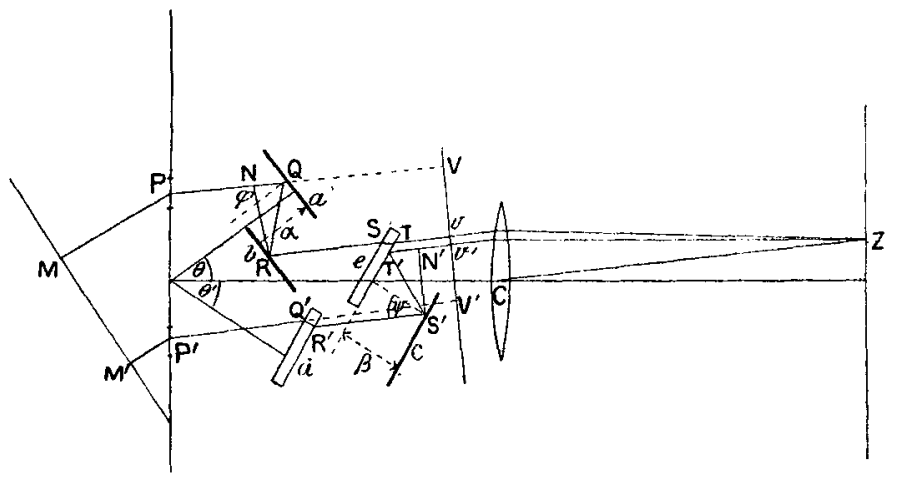

Suppose then that a plane wave of light whose front is $M M^{\prime}$ is incident upon the diaphragm. Let us break the wave up, as is usual, in the plane of the diaphragm. Let Z be a point on the screen whose cordinates are $(p, q)$ at which the intensity of light is required.

Then if $\mathrm{C}$ be the centre of the object-glass, the direction in which ravs $\mathrm{T} U, \mathrm{~T}^{\prime} \mathrm{U}^{\prime}$ must proceed in order to converge to $\mathrm{Z}$ after refraction is parallel to $\mathrm{CZ}$.

Hence $P Q, R S, T U, P^{\prime} Q^{\prime}, R^{\prime} S^{\prime}, T^{\prime} U^{\prime}$ are all parallel to $C Z$, and the direction-cosines of $\mathrm{CZ}$ are

$$
\frac{p}{\sqrt{p^{2}+q^{2}+b^{2}}}, \quad \frac{q}{\sqrt{p^{2}+q^{2}+b^{2}}}, \quad \frac{b}{\sqrt{p^{2}+q^{2}+b^{2}}} .
$$

I shall assume that the strips $e$ and $d$ are cut from the same plate and are of equal thickness. This will sensibly simplify the analysis, though, as I think, it would not materially influence the appearances if the strips were unequal.

If, however, we suppose them equal, we may neglect the presence of strips, as far as refraction is concerned, since clearly the retardation introduced is the same for all parallel rays.

If now $\mathrm{UU}^{\prime}$ be a plane perpendicular to $\mathrm{CZ}$, then, since we know that rays parallel to $\mathrm{TU}^{\prime}, \mathrm{T}^{\prime} \mathrm{U}^{\prime}$ converge to a focus at $\mathrm{Z}$, the only parts of the paths of the rays which can introduce a 
difference of phase are

$$
\begin{aligned}
& \mathrm{MP}+\mathrm{PQ}+\mathrm{QR}+\mathrm{RS}+\mathrm{TU} \text { for one stream, } \\
& \mathrm{M}^{\prime} \mathrm{P}^{\prime}+\mathrm{P}^{\prime} \mathrm{Q}^{\prime}+\mathrm{R}^{\prime} \mathrm{S}^{\prime}+\mathrm{S}^{\prime} \mathrm{T}^{\prime}+\mathrm{T}^{\prime} \mathrm{U}^{\prime} \text { for the other stream. }
\end{aligned}
$$

Produce $P Q, P^{\prime} Q^{\prime}$ to meet it in $V$ and $V^{\prime}$ and let $N, N^{\prime}$ be the feet of the perpendiculars from $R$ and $S^{\prime}$ on $P Q, T^{\prime} U^{\prime}$ respectively.

'i'hus we may take the change of phase as due to the retardation

$$
(\mathrm{MP}+\mathrm{PV})+(\mathrm{NQ}+\mathrm{QR})
$$

for diffraction at one slit, and to the retardation

$$
\left(\mathrm{M}^{\prime} \mathrm{P}^{\prime}+\mathrm{P}^{\prime} \mathrm{V}^{\prime}\right)+\left(\mathrm{S}^{\prime} \mathrm{T}^{\prime}+\mathrm{T}^{\prime} \mathrm{N}^{\prime}\right)
$$

for rays proceeding from the other slit.

The terms in the first brackets give us the expression which we had before, viz. :-

$$
-\left(\frac{p+u}{b}\right) x-\left(\frac{q+v}{b}\right) y+\text { const. }
$$

As to the other terms

$$
\mathrm{NQ}+\mathrm{QR}=\mathrm{QR}(1+\cos 2 \phi)=\alpha \frac{1+\cos 2 \phi}{\cos \phi}=2 \alpha \cos \phi,
$$

where $a$ is the distance between the mirrors $a, b$ and $\phi$ is the angle of incidence of any ray on these mirrors.

Similarly $S^{\prime} \Gamma^{\prime}+T^{\prime} N^{\prime}=2 \beta \cos \psi$, where $\beta$ is the distance between $e$ and $c$ and $\psi$ the angle of incidence of any ray npon $e$ and $c$.

Now if the mirrors $a$ and $b$ are inclined to the plane of the diaphragm at an angle $\theta, c, d, e$ at an angle $\left(-\theta^{\prime}\right)$, then

$$
\begin{aligned}
& \cos \phi=\frac{q \sin \theta+b \cos \theta}{\sqrt{p^{2}+q^{2}+b^{2}}}, \\
& \cos \psi=\frac{-q \sin \theta^{\prime}+b \cos \theta}{\sqrt{p^{2}+q^{2}+b^{2}}},
\end{aligned}
$$

To find the disturbance at $\mathrm{Z}$ we have

$$
\begin{aligned}
& \int_{a-k}^{a+k} d y \int_{-k}^{+h} d x \frac{A}{b \lambda} \sin \frac{2 \pi}{\lambda}\left(\frac{\lambda t}{\tau}+\frac{p+u}{b} x+\frac{q+v}{b} y-2 \alpha \cos \phi\right) \\
& +\int_{-a-k}^{-a+k} d \int_{-h}^{+h} d x \frac{A}{b \lambda} \sin \frac{2 \pi}{\lambda}\left(\frac{\lambda t}{\tau}+\frac{p+u}{b} x+\frac{q+v}{b} y-2 \beta \cos \psi\right),
\end{aligned}
$$


which, on being integrated, gives

$\frac{2 \mathrm{~A} b \lambda}{\pi^{2}(p+u)(q+v)} \sin \frac{2 \pi}{\lambda}-\frac{q+v}{b} k \sin \frac{2 \pi}{\lambda} \frac{p+u}{b} h \sin \frac{2 \pi}{\lambda}\left(\frac{\lambda t}{\tau}-\epsilon\right) \cos \frac{2 \pi \gamma}{\lambda}$,

where $-\epsilon-\gamma=-\frac{v+q}{b} a-2 \beta \cos \psi, \quad-\epsilon+\gamma=\frac{v+q}{b} a-2 \alpha \cos \phi$,

whence

$$
\begin{aligned}
& \epsilon=\alpha \cos \phi+\beta \cos \psi, \\
& \gamma=\frac{v+q}{b} a+\beta \cos \psi-\alpha \cos \phi .
\end{aligned}
$$

Hence the intensity I of light at $(p, q)$ is

$$
\frac{4 A^{2} b^{2} \lambda^{2}}{\pi^{4}(p+u)^{2}(q+v)^{2}} \sin ^{2} \frac{2 \pi}{\lambda} \frac{q+v}{b} v \sin ^{2} \frac{2 \pi}{\lambda} \frac{p+u}{b} h \cos ^{2} \frac{2 \pi \gamma}{\lambda}
$$

where $\gamma=\frac{v+q}{b} a+\frac{\beta \cos \theta^{\prime}-\alpha \cos \theta}{\sqrt{p^{2}+q^{2}+b^{2}}} b-\frac{(\beta \sin \theta+\alpha \sin \theta)}{\sqrt{p^{2}+q^{2}+b^{2}}} q$.

In the last term we may put $\frac{q}{\sqrt{p^{2}+q^{2}+b^{2}}}=\frac{q}{b}$, for if we went to a higher approximation, we should introduce cubes of $p / b$, $q / b$ which we have hitherto neglected.

If, further, we make $\beta \cos \theta^{\prime}=\alpha \cos \theta$, which can always be managed without difficulty, the second term, which would contain squares on expansion, disappears and we have

$$
\begin{aligned}
\gamma & =\frac{(v+q) a-\left(\beta \sin \theta^{\prime}+\alpha \sin \theta\right) q}{b} \\
& =\left\{v a+q\left(a-\left(\beta \sin \theta^{\prime}+\alpha \sin \theta\right)\right)\right\} / b .
\end{aligned}
$$

This gives fringes of breadth $b \lambda / 2\left(a-\left(\beta \sin \theta^{\prime}+a \sin \theta\right)\right)$. These may be reckoned from the bright fringe $\gamma=0 ; i$. e.

$$
q_{0}=\frac{-v a}{a-\left(\beta \sin \theta^{\prime}+\alpha \sin \theta\right)} .
$$

The visibility of the fringes for a single source will, as before, not be affected by changing $a$ : for a second source the origin of the fringes is given by

$$
q_{0}^{\prime}=-v^{\prime} a /\left(a-\left\{\beta \sin \theta^{\prime}+\alpha \sin \theta\right\}\right) .
$$

and if the visible rectangles overlap, there will be a sensible diminution of the fringe appearance whenever

where $n$ is an integer;

$$
q_{0}-q_{0}^{\prime}=\left(n+\frac{1}{2}\right) b \lambda / 2\left\{a-\left(\beta \sin \theta^{\prime}+\alpha \sin \theta\right)\right\}
$$

i.e.

$$
v^{\prime}-v=\text { an odd multiple of } b \lambda / 4 a,
$$

the condition previously found. 
One further point should be noticed : if $a$ be very nearly equal to $\beta \sin \theta^{\prime}+\alpha \sin \theta$ the fringes become too broad to be observed, whatever the source may be.

Fig. 5.

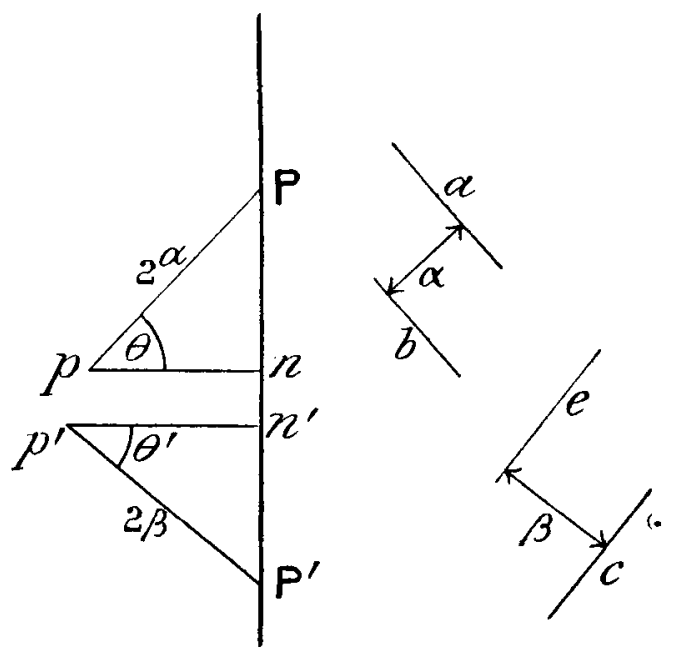

To see the physical meaning of this condition, and also of the condition $\beta \cos \theta^{\prime}=\alpha \cos \theta$, we notice that a point source of light $\mathrm{P}$ at the centre of one of the slits appears after reflexion at the two mirrors $a, b$, to be at $p$, where $\mathrm{P} p$ is equal to twice the distance between the mirrors and is perpendicular to their plane (fig. 5). Hence the double reflexion removes the image of the slit a distance $2 a \cos \theta$ behind the diaphragm and $2 a \sin \theta$ closer to the centre. In the same way the image of the other slit is brought $2 \beta \cos \theta^{\prime}$ behind the diaphragm and $2 \beta \sin \theta^{\prime}$ nearer the centre.

Our condition $\beta \cos \theta^{\prime}=a \cos \theta$ therefore means that the images of the two slits must be in the same plane parallel to the plane of the diaphragm itself, and our second condition shows that they must be some distance apart.

To find the minimum of this distance, remember that the fringes will be invisible if the distance between successive maxima exceeds the vertical dimension of the visible rectangle : in other words, if

$$
\begin{gathered}
b \lambda / 2\left(a-\left(\beta \sin \theta^{\prime}+\alpha \sin \theta\right)\right)>b \lambda / k, \\
\quad \text { or distance in question }<k,
\end{gathered}
$$

which means that the centre of the image of either slit must 


\section{Mr. L. N. G. Filon on certain Diffraction Fringes}

be outside the other. These two points must be carefully borne in mind in adjusting the instruments.

When this, however, is done, we see that Michelson's assertions are confirmed, and that when we increase the aperture of the telescope in this way, the results obtained are of the same character as when the slits are placed directly in front of the object-glass.

5. Let us now proceed to consider an extended source, which we shall suppose for simplicity to be of uniform intensity. foru

The intensity at a point $(p, q)$ on the screen will be of the

$$
\left.\mathrm{I}=\mathrm{A}^{2} \frac{64 h^{2} k^{2}}{b^{2} \lambda^{2}} \iint \frac{\sin \frac{2 \pi k(q+v)}{b \lambda}}{\left(\frac{2 \pi k(q+v)}{b \lambda}\right)} \cdot \frac{\sin \frac{2 \pi h(p+u)}{b \lambda}}{\left(\frac{2 \pi h(p+u)}{b \lambda}\right)}\right\}^{2} \cos ^{2} \frac{2 \pi a(q+v)}{v \lambda} d u d v
$$

the integral being taken all over the geometrical image of the extended source.

We have now three cases to consider.

(a) When the angular dimensions of the source are large compared with $\lambda / h$.

(b) When the angular dimensions of the source are small compared with $\lambda / h$.

(c) When the angular dimensions of the source are neither large nor small compared with $\lambda / h$.

I et us begin with case $(a)$. Then, if we consider a point inside the geometrical image, the two limits for $u$ will be very large, except where the vertical through the point cuts the image ; the quantity

$$
\left\{\frac{\frac{\sin \frac{2 \pi h(p+u)}{b \lambda}}{2 \pi h(p+u)}}{b \lambda}\right\}^{2}
$$

being insensible for all points outside a thin strip (shaded in the figure) having for its central line the line through $p, q$ perpendicular to the slits.

We may therefore, in integrating with regard to $u$, replace the limits by $\pm \infty$, and then integrate with regard to $v$ along the chord of the image perpendicular to the slits. 
Fig. 6.

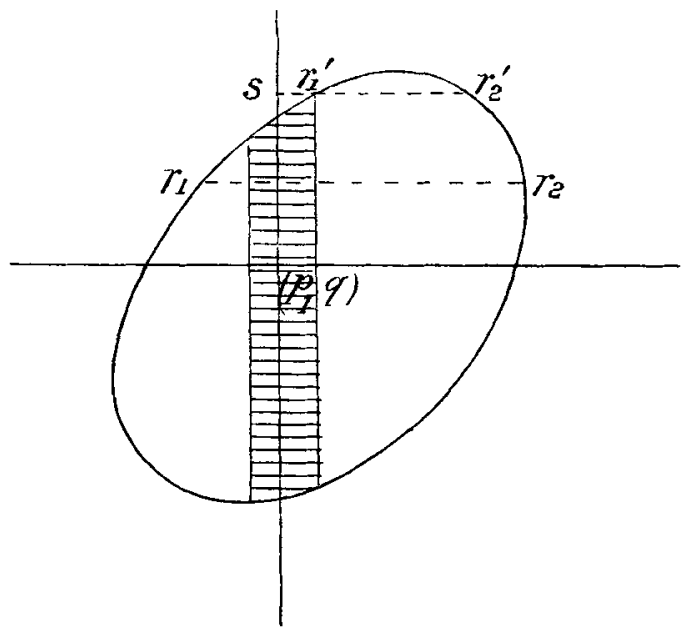

Hence, remembering that

it follows that

$$
\int_{-\infty}^{\infty} \frac{\sin ^{2} x}{x^{2}} d x=\pi
$$

$$
\mathrm{I}=\frac{32 \mathrm{~A}^{2} h k^{2}}{b \lambda}\left\{\frac{\sin \frac{2 \pi k}{b \lambda}(q+v)}{\frac{2 \pi k}{b \lambda}(q+v)}\right\}^{2} \cos ^{2} \frac{2 \pi a}{b \lambda}(q+v) d v .
$$

Now if the angular dimensions of the source of light be large compared with $\lambda / k$, the limits of integration with regard to $v$ may be made infinite. In this case the intensity I

$=\frac{8 \mathrm{~A}^{2} h k^{2}}{b \lambda} \int_{-\infty}^{\infty} d v\left\{\sin ^{2} \frac{2 \pi}{b \lambda}(a+k)(q+v)+\sin ^{2} \frac{2 \pi}{b \lambda}(a-k)(q+v)\right.$

$$
\left.-2 \sin ^{2} \frac{2 \pi a}{b \lambda}(q+v)+2 \sin ^{2} \frac{2 \pi k}{b \lambda} \cdot(q+v)\right\} \div\left(\frac{2 \pi k(q+v)}{b \lambda}\right)^{2}
$$

$=\frac{4 \mathrm{~A}^{2} h}{\pi}\left[\{(a+k)+(a-k)-2 a+2 k\} \int_{-\infty}^{+\infty} \frac{\sin ^{2} x}{x^{2}} d x\right]$

$=8 \mathrm{~A}^{2} h k=$ constant.

This result shows us that if the dimensions of the seurce exceed a certain limit, no diffraction-fringes exist at all, at least near the centre of the image. Next let the angular dimensions

Phil. Mag. S. 5. Vol. 47. No. 288. May 1899. 2 I 
454 Mr. L. N. G. Filon on eertain Diffraction Fringes

of the source be less than $\frac{\lambda}{12 k}$, then throughout the integration $\frac{2 \pi k}{b \lambda}(q+v)$ is less than $\pi / 6$ numerically.

But

$$
\frac{\sin ^{2} \frac{\pi}{6}}{\pi^{2} / 36}=\frac{9}{\pi^{2}},
$$

and differs but little from unity.

We may therefore in this case write

$$
\frac{\sin ^{2} \frac{2 \pi k}{b \lambda}(q+v)}{\left(\frac{2 \pi k}{b \lambda}(q+v)\right)^{2}}=1
$$

throughout the range of integration.

If now the limits be $v_{1}$ and $v_{2}$ we have

$$
\begin{aligned}
\mathrm{I} & =\frac{32 \mathrm{~A}^{2} h k^{2}}{b \lambda} \int_{v_{2}}^{v_{1}}\left(\frac{1}{2}+\frac{\pi}{2} \cos \frac{4 \pi a(q+v)}{b \lambda}\right) d v \\
& =\frac{4 \mathrm{~A}^{2} h k^{2}}{\pi a} \int_{v_{2}}^{v_{1}}\left(1+\cos \frac{4 \pi a(q+v)}{b \lambda}\right) d\left(\frac{4 \pi a(q+v)}{b \lambda}\right) \\
& =\frac{4 \mathrm{~A}^{2} / / k^{2}}{\pi a}\left\{\frac{4 \pi a}{b \lambda}\left(v_{1}-v_{2}\right)+\sin \frac{4 \pi a\left(q+v_{1}\right)}{b \lambda}-\sin \frac{4 \pi a\left(q+v_{2}\right)}{b \lambda}\right\} \\
& =\frac{4 \mathrm{~A}^{2} h k^{2}}{\pi a}\left\{\frac{4 \pi a\left(v_{1}-v_{2}\right)}{b \lambda}+2 \sin \frac{2 \pi a\left(v_{1}-v_{2}\right)}{b \lambda} \cos \frac{4 \pi a\left(q+\frac{1}{2}\left(v_{1}+v_{2}\right)\right)}{b \lambda}\right\} .
\end{aligned}
$$

Let $2 c=$ length of chord through the point perpendicular to the direction of the slits, then

$$
2 c=v_{1}-v_{2},
$$

and let $v_{0}=$ coordinate of the mid-point of this chord. Then

$$
\mathrm{I}=\frac{8 \mathrm{~A}^{2} h k^{2}}{\pi a}\left\{\frac{4 \pi a c}{b \lambda}+\sin \frac{4 \pi a c}{b \lambda} \cos \frac{4 \pi a\left(q+v_{0}\right)}{b \lambda}\right\} .
$$

The fringes therefore disappear when

Their visibility is

$$
\frac{4 \pi a c}{b \lambda}=s \pi
$$

$$
\sin \frac{4 \pi a c}{b \lambda} / \frac{4 \pi a c}{b \lambda}
$$


and is a maximum when

$$
\frac{4 \pi a c}{b \bar{\lambda}}=\tan \frac{4 \pi a c}{b \lambda} ;
$$

but the most visible fringes correspond to the early maxima.

This form agrees exactly with the formula given by Mr. Michelson for a uniformly illuminated segment of a straight line perpendicular to the slits. We see, however, that, provided the conditions stated be fulfilled, it, is applicable to a source of any shape.

The most general form of the fringes is given by

$$
q+\frac{1}{2}\left(v_{1}+v_{2}\right)=\text { const., }
$$

and therefore consists of lines parallel to the locus of middle points of chords at right angles to the slits. These will be straight lines in the case of a rectangular, circular, or elliptic source. Here, however, a new difficulty presents itself. For the rectangular source $v_{1}-v_{2}$ will be constant, whatever chord perpendicular to the slits we may select. Fringes will therefore appear and disappear as a whole.

But for a circular or elliptic source, $v_{1}-v_{2}$ varies as we pass from chord to chord. Thus the maxima will be invisible for some chords when they are most visible for others and conversely. Hence, whatever be the distance between the slits, it appears at first as it we might always expect a mottled appearance.

But in the case of a circular or elliptic sonree the length of the chord varies extremely slowly near the centre and there fringes will be visible, the length of the chord being practically constant. The mottled appearance, on the other hand, will predominate as we approach the sides.

6 . Consider now case $(b)$ and let tha dimensions of the source be so small that, for any point sufficiently close to the centre of the inage $\frac{2 \pi h(p+u)}{b \lambda}$ is a small angle throughout the range of integration.

[For points not near the centre of the image the illumination will be very small and the appearances are comparatively unimportant.]

For a point distant $<\frac{\lambda b}{24 h}$ from the centre of the image, we may put, as in previous reasoning,

$$
\left\{\frac{\sin \frac{2 \pi k}{b \lambda}(q+v)}{\frac{2 \pi k}{b \lambda}(q+v)} \frac{\sin \frac{2 \pi h}{b \lambda}(p+u)}{\frac{2 \pi h}{b \lambda}(p+u)}\right\}^{2}=1
$$


456 Mr. L. N. G. Filon on certain Diffraction Fringes

all over the range of integration, whence

$$
\begin{aligned}
\mathrm{I} & =\frac{32 \mathrm{~A}^{2} k^{2} h^{2}}{b^{2} \lambda^{2}} \iint\left(1+\cos \frac{4 \pi a(q+v)}{b \lambda}\right) d u d v \\
& =\frac{32 \mathrm{~A}^{2} k^{2} h^{2}}{b^{2} \lambda^{2}}\left(\Omega+\cos \frac{4 \pi a q}{b \lambda} \int u \cos \frac{4 \pi a v}{b \lambda} d v-\sin \frac{4 \pi a q}{b \lambda} \int u \sin \frac{4 \pi a v}{b \lambda} d v\right) \\
& =\frac{32 \mathrm{~A}^{2} k^{2} h}{b^{2} \lambda^{2}}\left(\Omega+\mathrm{R} \cos \left(\frac{4 \pi a q}{b \lambda}+\phi\right)\right),
\end{aligned}
$$

where $\Omega=$ total area of the image,

$$
\mathrm{R} \cos \phi=\int u \cos \frac{4 \pi \alpha v}{b \lambda} d v, \quad \mathrm{R} \sin \phi=\int u \sin \frac{4 \pi a v}{b \lambda} d v,
$$

the integrals being taken all over the image. 'The visibility $=\mathrm{R} / \Omega$ and therefore vanishes when $\mathrm{R}$ vanishes.

In the case of a circular source we find

$$
\phi=0, \quad \mathrm{R}=\text { (some non-vanishing factor), } \mathrm{J}_{1}\left(\frac{4 \pi a r}{b \lambda}\right),
$$

where $J_{1}$ is the Bessel's function of order unity and $r$ is the radius of the image, so that $r / b$ is the angular radins of the source. The dark fringes are given by

$$
\frac{4 \pi a q}{b \lambda}=(2 s+1) \pi,
$$

$q$ being measured from the centre of the source. The fringes are parallel to the slits and disappear whenever

$$
\mathrm{J}_{1}\left(\frac{4 \pi a r}{b \lambda}\right)=0 .
$$

This result agrees with the one given by Michelson for any circular source. We see that it only holds provided the dimensions of the source do not exceed a certain limit.

In the case of an elliptic source $\phi=0$ also, and $R$ is not altered by any sliding of the image parallel to the direction of the slits. Hence we may replace the oblique ellipse by one having its principal axis parallel and perpendicular to the slits, the values of the semi-axes being $d$ and , where $d==$ length of semi-diameter of original image parallel to the siits, $\varpi=$ length of perpendicular from the centre upon the parallel tangent. We find without difficulty: 


$$
\begin{aligned}
\mathrm{R} & =\int \cos \frac{4 \pi a v}{b \lambda} u d v \text { over the image } \\
& =4 \int_{0}^{\sigma \pi} \frac{d}{\varpi} \sqrt{\sigma^{2}-v^{2}} \cos \frac{4 \pi a v}{b \lambda} d v=\frac{d \varpi}{2} \cdot \frac{b \lambda}{a \varpi} \mathrm{J}_{1}\left(\frac{4 \pi a \varpi}{b \lambda}\right) \\
& =\Omega\left(\frac{b \lambda}{2 \pi a \varpi}\right) \mathrm{J}_{1}\left(\frac{4 \pi a \varpi}{b \lambda}\right) .
\end{aligned}
$$

The visibility is therefore

and vanishes whenever

$$
2 \mathrm{~J}_{1}\left(\frac{4 \pi a \varpi}{b \lambda}\right) /\left(\frac{4 \pi a \varpi}{b \lambda}\right)
$$

$$
\mathrm{J}_{1}\left(\frac{4 \pi a \varpi}{b \lambda}\right)=0 \text {. }
$$

Hence we see that, for an elliptic source, if $\rho=$ length of semidiameter perpendicular to the slits, $\sigma=$ length of perpendicular on the tangent parallel to the slits, then the fringes disappear when $\sin \frac{4 \pi a \rho}{b \lambda}=0$, if the angular dimensions are of order $\frac{1}{12} \frac{\lambda}{k}$ as indicated above, and when $J_{1}\left(\frac{4 \pi a \sigma}{b \lambda}\right)=0$, when the angular dimensions are less than $\frac{1}{12} \frac{\lambda}{h}$.

In the first case $\rho$ is the quantity which determines the disappearance of the fringes, in the second case $\omega$ : and further, we see that the validity of the formulæ is entirely dependent on the length and breadth of the slit, neither of which is considered by Mr. Michelson.

We may notice that the best results are obtained, in the first case when $h$ is large, in the second case when $h$ is small.

7. It remains to consider the intermediate case $(c)$. This does not perhaps present so much interest as the other two ; the first will generally correspond to the case of a planet, the second to that of a star, in astronomical observations.

In dealing with case $(c)$ we shall suppose the angular dimensions to be small, with regard to $\lambda / k$, but not with regard to $\lambda / h$. We may then write

$$
\begin{gathered}
I=\frac{6 \mathrm{~A}^{2} h^{2} k^{2}}{b^{2} \lambda^{2}} \iint\left\{\frac{\sin \frac{2 \pi k(q+v)}{b \lambda}}{\frac{2 \pi k(q+v)}{b \lambda}} \cdot \frac{\sin \frac{2 \pi h(p+u)}{b \lambda}}{\frac{2 \pi h(p+u)}{b \lambda}}\right\}^{2} \cos ^{2} \frac{2 \pi a(q+v)}{b \lambda} d u d v \\
=\frac{32 \mathrm{~A}^{2} h^{2} k^{2}}{l^{2} \lambda^{2}} \iint \frac{\sin ^{2} \frac{2 \pi h u}{b \lambda}}{\left(\frac{2 \pi h u}{b \lambda}\right)^{2}}\left(1+\cos \frac{4 \pi a(q+v)}{l \lambda}\right) d u d v,
\end{gathered}
$$


458 Mr. L. N. G. Filon on certain Diffraction Fringes

if we only consider the appearances along the line $p=0$, taken to pass through the centre of the image, which is assumed circular or elliptic.

Denoting $\frac{2 \pi h}{b \lambda}$ by $\mu$ and expanding, we get $\frac{\sin ^{2} \frac{2 \pi h u}{b \lambda}}{\left(\frac{2 \pi h u}{b \lambda}\right)^{2}}=1-\frac{1}{2} \cdot \frac{2^{2} \mu^{2} u^{2}}{3 !}+\ldots+\frac{(-1)^{r-1}(2 \mu u)^{2(r-1)}}{r \cdot(2 r-1) !}+\ldots$

whence

$$
\begin{array}{r}
I=\frac{32 A^{2} h^{2} k^{2}}{b^{2} \lambda^{2}}\left[\mathrm{~K}+\int\left(u-\frac{1}{2.3} \frac{(2 \mu)^{2} u^{3}}{3 !}+\ldots+\frac{(-1)^{r-1} \frac{(2 \mu)}{r(2 r-1)}}{{ }^{2(r-1)}} \frac{u^{2 r-1}}{(2 r-1) !}+\ldots\right)\right. \\
\left.\times \cos \frac{4 \pi u(q+v)}{b \lambda} d v\right]
\end{array}
$$

where

$$
\mathrm{K}=\iint \frac{\sin ^{2} \mu u}{\mu^{2} u^{q}} d u d v
$$

taken all over the source, and is essentially positive and independent of $a$ and $q$.

Now

$$
\int u^{2 r-1} \sin \frac{4 \pi a(v)}{b \lambda} d v=0
$$

for a circle or ellipse.

To find the cosine integral, C, we have, $d$ and $\approx$ having the same meaning as before,

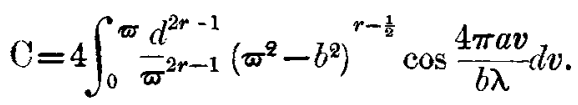

Put $v=\varpi \cos \theta$.

$$
\begin{aligned}
\mathrm{C} & =4 d^{2 r-1} \varpi \int_{0}^{\frac{\pi}{2}} \sin ^{2 r} \theta \cos \left(\frac{4 \pi a \varpi}{b \lambda} \cos \theta\right) d \theta \\
& =\frac{2 d^{2 r-1} \varpi 2^{r} \sqrt{\pi} \Gamma\left(r+\frac{1}{2}\right)}{\left(\frac{4 \pi a \varpi}{b \lambda}\right)^{r}} \mathrm{~J}_{r}\left(\frac{4 \pi a \varpi}{b \pi}\right) \\
& =\frac{2 d^{2 r-1} \varpi \pi(2 r-1) !}{2^{r-1}(r-1) !} \frac{\mathrm{J}_{r}\left(\frac{4 \pi a \varpi}{b \lambda}\right)}{\left(\frac{4 \pi a \varpi}{b \lambda}\right)^{r}}
\end{aligned}
$$


às applied to Micrometric Observations.

Hence:

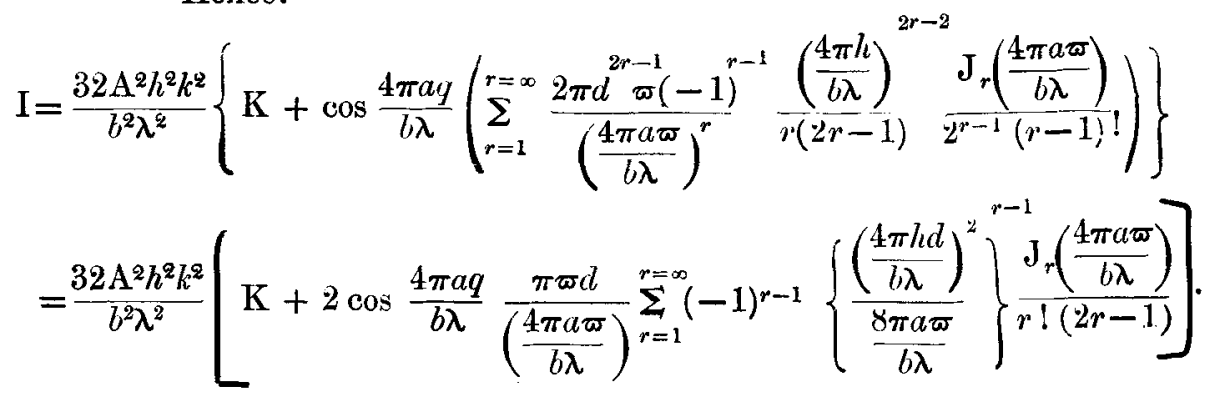

Denote by $\Omega$ the total area of the image and by $e$ the ratio

Then the visibility

$$
\left(\frac{4 \pi h d}{b \lambda}\right)^{2} /\left(\frac{8 \pi a \varpi}{b \lambda}\right)
$$

$$
\begin{aligned}
=\frac{2 \Omega}{\mathrm{K}} \frac{1}{\frac{4 \pi a \omega}{b \lambda}}\left\{\mathrm{J}_{1}\left(\frac{4 \pi a \varpi}{b \lambda}\right)\right. & -\frac{e}{6} \mathrm{~J}_{2}\left(\frac{4 \pi a \varpi}{b \lambda}\right)+\ldots \\
& \left.+(-1)^{r-1} \frac{e^{r-1}}{r !(2 r-1)} \mathrm{J}_{r}\left(\frac{4 \pi a \varpi}{b \lambda}\right)+\ldots\right\}
\end{aligned}
$$

The series for the visibility is absolutely convergent, because $\mathrm{J}_{n+1}(x) / J_{n}(x)$ decreases numerically without limit as $n$ increases without limit.

The roots of the equation

$$
J_{1}\left(\frac{4 \pi a \varpi}{b \lambda}\right)-\frac{e}{6} J_{2}\left(\frac{4 \pi a \varpi}{b \lambda}\right)+\ldots+(-1)^{r-1} \frac{e^{r-1}}{r !(2 r-1)} J_{r}\left(\frac{4 \pi a \varpi}{b \lambda}\right)+\ldots=0
$$

give the values of $a$ for which the visibility vanishes.

Notice, however, that $e$ contains $d$ and the length of the slit, so that the values obtained for $a$ will be functions of the horizontal diameter and of the length of the slit.

8. Besides enabling us to determine the angular distance of two point-sources and the radius of an extended source, Mr. Michelson's method allows us to detect and measure the ellipticity of a luminous disk.

Referring to the fornulæ for cases $(a)$ and $(b)$, the visibility vanishes when

$$
\sin \frac{4 \pi a \rho}{b \lambda}=0 \text { in case }(a)
$$

and when

$$
\mathrm{J}_{1}\left(\frac{4 \pi a \varpi}{b \lambda}\right)=0 \text { in case }(b)
$$


In either of these cases, if we rotate the slits about the axis of the telescope, without altering $a$, then if the source is elliptic, $\rho$ and $\sigma$ will vary, and the visibility of the fringes will vary.

Now suppose for a given position of the slits we vary $a$ until the visibility $=0$ for that position, and then rotate the slits and note the different inclinations for which it vanishes.

It will certainly vanish once again before a complete halfturn has been made, namely, when the slits make an angle with the direction of either axis of the ellipse equal to that which they made at first, but on the other side of the axis.

It may vanish more than once, but since the inclinations for which it vanishes are symmetrical with regard to the axes of the ellipse, there will usually be no difficulty in determining the directions of the axes.

Their lengths can then be determined by two observations of the disappearance of the fringes, one for each of the two positions of the slits which are perpendicular to an axis.

It must, however, be noticed that the accuracy of this method for measuring ellipticity decreases with the size of the source, inasmuch as the quantity which causes the alteration in the fringes is the difference, not the ratio of the semiaxes.

To get some idea of the sensitiveness of the method, let us estimate roughly the amount of ellipticity which could be detected in a disk of angular semi-diameter $10^{\prime \prime}$, taking the mean wave-length of light $5 \times 10^{-4} \mathrm{cms}$.

The visibility vanishes when $\sin \frac{4 \pi a \rho}{b \lambda}=0$, and will be quite sensible when $\sin \frac{4 \pi a \rho}{6 \lambda}=\frac{1}{2}$, say. Hence in order that we may be able to note a sensible difference of visibility in the fringes, we must have

$$
\frac{4 \pi a}{\lambda}\left(\frac{\rho_{1}-\rho_{2}}{b}\right)=\frac{\pi}{6} \text { at least; }
$$

Or.

$$
\frac{\rho_{1}-\rho_{2}}{\tilde{b}}=\frac{1}{2} \frac{1}{10^{8}}
$$

if $a$ be a little above 4 cms.

$\therefore$ difference of angular semi-axes $=\cdot 01$ (semi-diameter) $q . p .$, or the amount of ellipticity which can be detected $=\cdot 01$.

I have taken $\sin \frac{4 \pi a \rho}{b \lambda}=0$ as giving zero visibility, because this example will clearly fall under case $(a)$. 
9. Summing up the results obtained we see that:-

(1) It is possible by the observation of Michelson's interference-fringes to separate a double point-source, or detect breadth and ellipticity in a slightly extended source.

(2) But the distance between the two points, or the dimensions of the extended source, must lie within certain limits depending on the length and breadth of the slits $*$.

(3) The dimensions of the slits also considerably affect the general theory, the formulæ obtained not being identical with Michelson's. The law of appearance and disappearance of the fringes depends very largely on the distance between the points or the dimensions of the extended sonrce.

XLII. The Absorption of Water in Hot Glass. Second Paper. By Carl Barus $\dagger$.

1. ATER finishing my account $\ddagger$ of the action of hot A water on glass, observed in fine-bore capillary tubes, it seemed to me that the experiments made several years ago (1891) left questions of considerable interest outstanding. I refer in particular to a further examination of the contents of the capillary tubes. Certain evidence was to be obtained as to the occurrence of syrupy glass at $185^{\circ}$, solidifying to a firm glass when cold, the composition remaining unchanged except as to the water absorbed. Again, as it was improbable that volume-contraction would continue at the same rate indefinitely, the conditions of subsidence were to be determined, together with the effect of the elastic and viscous constants of the tube itself on the apparent volume contractions and compressibilities observed. Questions relative to the acceleration of the reaction at higher temperatures were to be held in view.

Finally, if the inferences drawn from data for capillary tubes are correct, it should be possible to obtain the fusible glass on a larger scale. Experiments in progress in this direction have proved quite successful, and will be described in a later paper. The present remarks are restricted to the experiences with capillary tubes.

2. The apparatus used was the same as that heretofore

* Since the above was read, a paper has appeared in the Comptes Rendus de l'Académie des Sciences for Nov. 28, 1898, dealing with the modifications in Michelson's formulx when we take into account the breadth of the slits. The author, M. Hamy, follows Michelson in not considering variations of intensity parallel to the slits. This, I think, accounts for his results not quite agreeing with mine.

+ Communicated by the Author.

$\ddagger$ Phil. Mag. (5) xlvii, p. 104, Jan. 1899. 